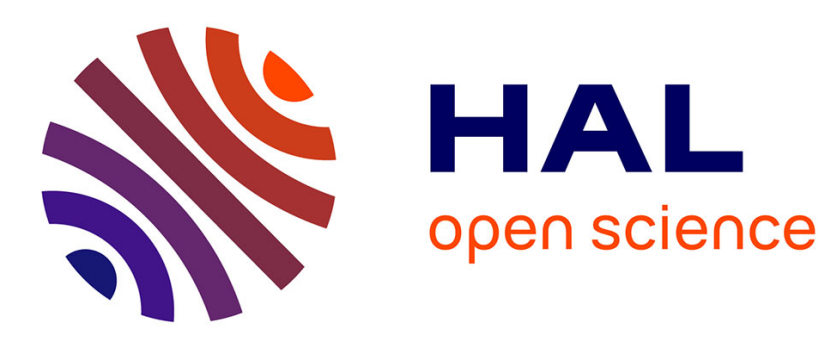

\title{
Note on Minimax Sliding Mode Control Design for Linear Systems
}

Sergiy Zhuk, Andrey Polyakov, Olexander Nakonechnyi

\section{To cite this version:}

Sergiy Zhuk, Andrey Polyakov, Olexander Nakonechnyi. Note on Minimax Sliding Mode Control Design for Linear Systems. IEEE Transactions on Automatic Control, 2016, 10.1109/TAC.2016.2612058 . hal-01371091

\section{HAL Id: hal-01371091 \\ https://hal.inria.fr/hal-01371091}

Submitted on 23 Sep 2016

HAL is a multi-disciplinary open access archive for the deposit and dissemination of scientific research documents, whether they are published or not. The documents may come from teaching and research institutions in France or abroad, or from public or private research centers.
L'archive ouverte pluridisciplinaire HAL, est destinée au dépôt et à la diffusion de documents scientifiques de niveau recherche, publiés ou non, émanant des établissements d'enseignement et de recherche français ou étrangers, des laboratoires publics ou privés. 


\title{
Note on Minimax Sliding Mode Control Design for Linear Systems
}

\author{
Sergiy Zhuk, Andrey Polyakov and Olexander Nakonechnyi
}

\begin{abstract}
The paper studies the problem of sliding mode control design for linear systems with incomplete and noisy measurements of the output and additive/multiplicative exogenous disturbances. First, we construct a linear minimax observer to have an estimate of the system's state with minimal worst-case error. Second, we establish the optimality of the constructed observer in the class of all observers represented by measurable functionals of the output. Finally, we propose an algorithm, generating continuous and discontinuous feedbacks, which steers the observer as close as possible to a given sliding hyperplane in finite time. The optimality (sub-optimality) of the designed feedbacks is proven for the case of bounded noises and additive (multiplicative) disturbances of $L^{2}$-class. The efficacy of the proposed algorithm is illustrated by a numerical example.
\end{abstract}

Index Terms-sliding mode control

\section{INTRODUCTION}

Sliding mode method was introduced more that 50 years ago (see [20] and references therein). Historically it was the first approach to robust control design. Indeed, it is well-known (see [7], [21], [18]) that sliding mode control is insensitive to a wide class of disturbances and uncertainties.

Robust output-based feedback control algorithms are required for many practical applications. The output-based sliding mode control design methodology is well-developed for linear systems (see, for example, [6], [7], [21], [18] and references therein). However, in practise, it is quite difficult to apply the state of the art sliding mode methods in the case of noisy measurements [19], [16] and/or mismatched disturbances, e.g. [8], [2], [15]. The aim of this paper is to propose a mathematically sound extension of the sliding mode control methodology allowing one to deal with the aforementioned cases efficiently. Specifically, we consider conventional (first order) sliding mode control principles and study the problem of observer-based sliding mode control design for a linear plant with additive/multiplicative exogenous disturbances and bounded (in $L^{2}$ ) deterministic measurement noises. Note that, in this case, it is impossible to ensure the ideal sliding mode due to noisy measurements. Instead, one can only design a control law $u$ providing the system motion as close as possible to the selected sliding surface. To design such $u$ we first construct a reachability set for the plant and then solve the following optimal control problem: find a feedback control $u$ steering the minimax center of the

S. Zhuk is with IBM Research, Server 3, Damastown Ind. Park, Dublin 15, Ireland, sergiy.zhukdie.ibm.com

A. Polyakov is with Inria Lille-Nord Europe, 40, avenue Halley, 59650 Villeneuve d'Ascq, France, andrey.polyakov@inria.fr

O. Nakonechnyi is with Taras Shevchenko National University of Kyiv, Ukraine, nakonechniy@unicyb.kiev.ua reachability set towards the sliding surface. We show that, for the case of noisy output measurements, the classical sliding mode control algorithm is just one of many possible solutions of the aforementioned optimal control problem (see Section IV), which, in fact, admits both continuous and discontinuous solutions in feedback form.

We rely upon the minimax state estimation framework [11], [3], [10] and duality argument [22], [23], [24] in order to construct the reachability set: the minimax state estimator or observer constructs a linear estimate of the plant's state with minimal worst-case error, provided the uncertain parameters (unknown initial condition, model disturbance, observation noise) have bounded $L^{2}$-energy. It turns out that (i) the state of the minimax observer coincides with the minimax center of the reachability set and (ii) the observer may be realized by a linear time-variant system taking the output of the original system as an input. The property (ii) allows us to apply linear separation principle and design the sliding mode control $u$ in the state space of the observer. Specifically, (i) guarantees that the state of the plant (with disturbances) is enclosed in the ellipsoid, which is centered at the state of the minimax estimator. Hence, if the control $u$ steers the observer's state towards a sliding surface, it follows that the actual state of the plant is guaranteed to be in a vicinity of the sliding surface, provided $u$ has been applied to the plant.

We prove that the minimax observer (in the form of a linear functional of outputs), used in this paper, is optimal among all observers represented by measurable functionals of outputs. Thus, at least theoretically, the proposed control design can not be further improved by using observers realized by non-linear functionals of outputs. The latter agrees with the numerical comparisons [25] of the control laws based on the minimax observer and the fixed-time feedbacks [13] based on the second order sliding mode (2-sm) observer [5], which is nonlinear. We refer the reader to [18], [7], [21], [16] for further information on sliding mode observers. Finally, we do a numerical comparison of the proposed algorithm and the $H^{\infty}$-observer based sliding mode control in Section V.

A preliminary version of this paper appeared in [26]. The key differences with respect to [26] are as follows: we show that the minimax observer in the form of a linear functional of observations is optimal for a wide class of observers (Proposition 1), show that optimal sliding mode control solves a minimax control problem (Proposition 1) and prove the existence of the sub-optimal control for the case of multiplicative disturbances (Proposition 3).

The paper is organized as follows. The next section presents the problem statement and basic assumptions. The minimax 
observer for linear systems is discussed in Section III. The problem of control design is studied in Section IV. Next the numerical simulation results and conclusions are provided. The proofs of all propositions are given in the appendix.

Throughout the paper the following notations are used: $\mathbb{R}$ is the set of real numbers; $\|x\|=\sqrt{x_{1}^{2}+\ldots+x_{n}^{2}}$ for $x=\left(x_{1}, \ldots, x_{n}\right)^{\top} \in \mathbb{R}^{n} ; L_{(a, b)}^{2}\left(\mathbb{R}^{s}\right)$ denotes Lebesgue space of squared integrable functions mapping $(a, b)$ to $\mathbb{R}^{s}$; the sign function is denoted by sign, i.e. $\operatorname{sign}[\rho]=1$ for $\rho>0$, $\operatorname{sign}[\rho]=-1$ for $\rho<0$ and $\operatorname{sign}[0] \in[-1,1]$.

\section{Problem StATEMENT}

Let us consider the following linear system:

$$
\begin{aligned}
& \dot{x}(t)=A x(t)+(B+p(t) E) u(t)+D d(t), x(0)=x_{0} \\
& y(t)=C x(t)+w(t), t \in[0, T),
\end{aligned}
$$

where $0<T<+\infty, x(t) \in \mathbb{R}^{n}$ is the vector of system state, $u(t) \in \mathbb{R}^{m}$ is the vector of the control inputs, $y(t) \in \mathbb{R}^{k}$ is the measured output, $d \in L_{(0, T)}^{2}\left(\mathbb{R}^{r}\right)$ and $p \in L_{(0, T)}^{2}(\mathbb{R})$ are unknown external disturbances; $w \in L_{(0, T)}^{2}\left(\mathbb{R}^{k}\right)$ is unknown deterministic measurement noise, the matrices $A \in$ $\mathbb{R}^{n \times n}, B, E \in \mathbb{R}^{n \times m}, D \in \mathbb{R}^{n \times r}, C \in \mathbb{R}^{k \times n}$ are assumed to be known and time-invariant.

We study this system under the standard assumptions: namely, the pair $\{A, C\}$ is observable and the pair $\{A, B\}$ is controllable. We do not restrict matrices $E$ and $D$ (recall that for $D \in \operatorname{range}(B)$ and $E=B$ the exogenous disturbances become matched [21], [7]).

We further assume that $x_{0}, p, d, w$ are uncertain and deterministic, and satisfy the following inequality:

$$
x_{0}^{\top} P_{0}^{-1} x_{0}+\int_{0}^{T} w^{\top}(\tau) R w(\tau)+d^{\top}(\tau) Q d(\tau)+\frac{p^{2}(\tau)}{\alpha} d \tau \leq 1,
$$

where $P_{0} \in \mathbb{R}^{n \times n}, R \in \mathbb{R}^{k \times k}$ and $Q \in \mathbb{R}^{r \times r}$ are given symmetric positive definite matrices, $\alpha>0$.

The classical sliding mode control problem is (see, [21], [7]) to find a feedback control law $u$ which steers the state of (1) towards a given linear hyperplane:

$$
F x=0, \quad F \in \mathbb{R}^{m \times n}, \quad \operatorname{det}(F B) \neq 0,
$$

and guarantees that the state does not leave this plane. It is worth noting [21], [7] that the condition $\operatorname{det}(F B) \neq 0$ is necessary for existence of a control law, which ensures sliding mode on the surface $F x=0$. The problem of optimal selection of the matrix $F$ in order to minimize the effects of disturbances is well studied in the literature (see, e.g. [2], [15]). We assume that some matrix $F$ is selected and we need to realize the reaching phase, i.e. to find the control law $u$ such that $F x(T)=0$. The considered problem can be equivalently formulated as $\|F x(T)\| \rightarrow$ min subject to (1) - (3). Indeed, obtaining a solution of this optimization problem with zero value of the cost functional guarantees the successful reaching of the sliding surface. However, due to unknown measurement noises and uncertain system disturbances, the exact reaching $F x(T)=0$ cannot be guaranteed. In order to construct a feedback control, which will realize the motion of the system as close as possible to the given surface provided the parameters $x_{0}, p, d, w$ are unknown and satisfy (3), we consider a minimax version of the classical Mayer optimal control problem $\|F x(T)\| \rightarrow \min _{u}$ (see, for example, [17], [1]):

$$
\begin{gathered}
\sup _{\left(x_{0}, d, p, w\right) \in \Omega_{T}}\|F x(T)\| \rightarrow \min _{u} \\
\text { s.t. (1) - (2) }
\end{gathered}
$$

where $\Omega_{T}$ is the ellipsoidal set composed of all $x_{0}, d, p, w$ verifying (3). The main goal of this technical note is to find (sub)optimal solution to the minimax optimization problem (4) in the feedback form. Note that we restrict our considerations to causal feedback laws, i.e. $u(t)$ may depend only on measurements $y(\tau)$ with $\tau \in[0, t]$.

\section{Minimax OBSERVER DESIGN}

According to the classical methodology of the sliding mode control design, the precise knowledge of the so-called sliding variable $\sigma(t):=F x(t)$ is required in order to ensure the motion of the system (1) on the surface $F x=0$. We stress that this information is not available as the given output $y(t)$ is incomplete and noisy. In this situation, the best available information about the value of $F x(t)$ can be represented by a minimax estimate of $x(t)$. Following [23] we introduce

Definition 1: Assume that $l \in \mathbb{R}^{n}$ and $\widehat{U} \in L_{\left(0, t^{*}\right)}^{2}\left(\mathbb{R}^{k}\right)$. A linear functional $\widehat{\mathcal{U}}_{l}(y):=\int_{0}^{t^{*}} \widehat{U}^{\top}(\tau) y(\tau) d \tau$ is called a minimax estimate of $l^{\top} x\left(t^{*}\right)$ iff

$$
\sigma\left(\widehat{U}, l, t^{*}\right) \leq \sigma\left(U, l, t^{*}\right), \quad \forall U \in L_{\left(0, t^{*}\right)}^{2}
$$

where $\sigma\left(\widehat{U}, l, t^{*}\right):=\sup _{\left(x_{0}, d, p, w\right) \in \Omega_{t^{*}}}\left|l^{\top} x\left(t^{*}\right)-\widehat{\mathcal{U}}_{l}(y)\right|$ and the set $\Omega_{t^{*}}$ is defined by (3) with $T=t^{*}$.

The number $\hat{\sigma}\left(l, t^{*}\right):=\sigma\left(\widehat{U}, l, t^{*}\right)$ is called the minimax estimation error.

Proposition 1: Let $\mathcal{G}$ denote a set of all continuous mappings of $L_{\left(0, t^{*}\right)}^{2}$ into $\mathbb{R}$ and let $g \in \mathcal{G}$. It then follows that

$$
\sigma\left(\widehat{U}, l, t^{*}\right)=\inf _{g \in \mathcal{G}} \sup _{\left(x_{0}, d, p, w\right) \in \Omega_{t^{*}}}\left|l^{\top} x\left(t^{*}\right)-g(y)\right| .
$$

In other words, the worst-case estimation error of any continuous mapping $g$, defined by the $\sup \{\}$ in (5), cannot be less than the minimax error of the linear functional $\widehat{\mathcal{U}}_{l}(y)$, which has minimal worst-case estimation error $\hat{\sigma}$. We refer the reader to Appendix for proofs of all the propositions.

Proposition 2: Let $\hat{x}(t) \in \mathbb{R}^{n}$ be the solution of the $O D E$ :

$$
\left\{\begin{array}{l}
\frac{d \hat{x}(t)}{d t}=A \hat{x}(t)+P(t) C^{\top} R(y(t)-C \hat{x}(t))+B u(t), \\
\hat{x}(0)=0
\end{array}\right.
$$

where $P(t) \in \mathbb{R}^{n \times n}$ is the solution to the differential Riccati equation:

$$
\begin{aligned}
\dot{P}(t)= & A P(t)+P(t) A^{\top}+D Q^{-1} D^{\top}+ \\
& \alpha E u(t) u^{\top}(t) E^{\top}-P(t) C^{\top} R C P(t),
\end{aligned}
$$

Then $\widehat{\mathcal{U}}_{l}(y)=l^{\top} \hat{x}\left(t^{*}\right), \hat{\sigma}\left(l, t^{*}\right)=\left(l^{\top} P\left(t^{*}\right) l\right)^{\frac{1}{2}}$.

A priori reachability set. Note that $\forall t^{*} \in[0, T]$ the definition of the minimax error $\widehat{\sigma}$ implies

$$
l^{\top}\left(x\left(t^{*}\right)-\hat{x}\left(t^{*}\right) \leq\left(l^{\top} P\left(t^{*}\right) l\right)^{\frac{1}{2}}, \forall l \in \mathbb{R}^{n} .\right.
$$


On the other hand, if (8) holds true for any $l \in \mathbb{R}^{n}$ and $t \in$ $[0, T]$ then, (see, for example, [10]) it follows that

$$
x(t) \in\left\{z \in \mathbb{R}^{n}: z=\hat{x}(t)+e, \quad e^{\top} P^{-1}(t) e \leq 1\right\},
$$

i.e. the state vector $x(t)$ belongs to the ellipsoid centered at $\hat{x}(t)$ with axes defined by the eigenvectors of $P^{-1}(t)$. It is worth noting that the estimate (9) is sharp, namely, for any $t \in[0, T]$ and for any $e_{*} \in \mathbb{R}^{n}$ such that $e_{*}^{\top} P^{-1}(t) e_{*} \leq 1$ there exist $\left(x_{0}^{*}, d^{*}, w^{*}, p^{*}\right) \in \Omega_{T}$ such that the equality $x(t)=\hat{x}_{u}(t)+e^{*}$ holds for some $x(t)$ satisfying (1)-(2). In fact, (9) describes the worst-case realisation of the reachability set of (1), i.e., it takes into account all $\left(x_{0}, d, w, p\right) \in \Omega_{T}$. The estimate (9) can be further improved if one "conditions" the reachability set on the output signal $y$ (see [10]). We do not use this result, however, as our controller design is supposed to be implemented without prior knowledge of $y$. Finally, we stress that the matrix $P$ does not depend on the control parameter $u$, provided $E=0$. This suggests to design the controller $u$ as a function of the center of the ellipsoid, $\hat{x}$ (see Section IV).

Stability of the observer for the case $T=+\infty$. If $E=0$ then, for an observable (or just detectable) pair $\{A, C\}$ there exist a unique symmetric matrix $P^{\infty} \geq 0$ such that $\lim _{t \rightarrow \infty} P(t)=P^{\infty}, A-P^{\infty} C^{\top} R C$ is stable, and $P^{\infty}$ solves the algebraic Riccati equation:0 $=A P+P A^{\top}+$ $D Q^{-1} D^{\top}-P C^{\top} R C P$. The proof of this well-known fact can be found in [17]. Thus, the inclusion (9) holds true for any $t \geq 0$. Moreover, if one sets $P(t):=P^{\infty}$, then, $\hat{x}$ solves a stable system (6), and, asymptotically, all the states $x(t)$ are contained in the ellipsoid defined by (9). In the case of multiplicative disturbances $(E \neq 0)$ the separation principle does not apply and stability of the observer depends on the control law $u$ (see Proposition 3).

We stress that $d \in L_{(0, T)}^{2}$ "vanish on infinity", i.e., the measure of the set $\{t>T:\|d(t)\|>0\}$ tends to 0 provided $T \rightarrow+\infty$. However, it is not hard to modify the observer in order to handle the case of $d \notin L_{(0, T)}^{2}$. For instance, the external perturbation $d$ may contain an unknown absolutely continuous function $v$ such that $v(0)^{\top} S v(0) \leq 1$ and $\lim _{t \rightarrow \infty} v(t) \neq 0$. In this case we introduce an augmented system $\dot{x}(t)=A x(t)+(B+p(t) E) u(t)+D d(t)+D v(t)$, $\dot{v}(t)=\xi(t)$ and $v(0)=v_{0}$, and extend (3) by adding $v_{0}^{\top} S v_{0}$, and placing the term $\xi^{\top}(s) W \xi(s)$ under the integral sign to account for $v_{0}$ and the unknown function $\xi \in L_{(0, T)}^{2}\left(\mathbb{R}^{r}\right)$ respectively. The matrices $W, S$ are assumed to be symmetric and positive definite. Then we set $x_{n e w}:=(x, v)^{\top}$, $A_{\text {new }}=\left(\begin{array}{cc}A & D \\ 0 & 0\end{array}\right), C_{\text {new }}=\left(\begin{array}{ll}C & 0\end{array}\right)$ and apply Proposition 2 for any $T<+\infty$. Now, to ensure stability of the observer for $T=+\infty$ we should assume that the pair $\left\{A_{n e w}, C_{n e w}\right\}$ is observable and use the arguments of the previous paragraph. Note that this condition is sufficient for the so-called strong observability [18] of the augmented system in the noise-free case $(w=0)$.

\section{Control Design}

\section{A. The case of additive disturbances: $E=0$}

Denote the sliding variable by $\sigma=F x$. By using (9) (recall from Section III that (9) is sharp) we derive

$$
\sigma(t)=F x(t)=\hat{\sigma}(t)+F e(t), \quad e^{\top}(t) P^{-1}(t) e(t) \leq 1,
$$

where $\hat{\sigma}(t)=F \hat{x}(t)$ and $\hat{x}$ satisfies (6). Moreover, the equation

$$
\frac{d \hat{\sigma}}{d t}=F A \hat{x}+F P(t) C^{\top} R(y(t)-C \hat{x})+F B u(t) .
$$

defines dynamics of the variable $\hat{\sigma}$.

Theorem 1: Any control $u$ which verifies the equality:

$$
\hat{\sigma}(T)=0
$$

is a solution to the minimax control problem (4) for $E=0$.

Let us consider the classical sliding mode control [21]

$$
u_{s m}(t)=-(F B)^{-1} K(t) \operatorname{sign}[\hat{\sigma}(t)], \quad K(t)>0 .
$$

then it follows that

$$
\frac{d \hat{\sigma}}{d t}=F A \hat{x}+F P(t) C^{\top} R(y(t)-C \hat{x})-K(t) \operatorname{sign}[\hat{\sigma}(t)]
$$

and taking into account $\hat{x}(0)=0$ we derive that (11) holds true if the relay feedback gain $K$ is selected as follows:

$$
K(t)=\left\|F A \hat{x}+F P(t) C^{\top} R(y(t)-C \hat{x})\right\|+\mu, \quad \forall \mu>0 .
$$

Therefore, the conventional sliding mode control is the solution of the minimax control problem (4) for $E=0$.

The right-hand side of the closed-loop sliding mode control system is discontinuous with respect to the extended state variables $(x, \hat{x}, P)$. Its solution is understood in the sense of Filippov. Since the system is affine with respect to control input, then other definitions (Utkin and Aizerman-Pyatnickii) are equivalent to Filippov one (see, for example, [19], [14]). In particular, Utkin's method applied to the closed-loop system (1), (7), (6), (12), (13) gives the equivalent control of the form (14). Therefore, to fulfill the condition (11), the continuous control can be designed:

$$
u_{e q}(t)=-(F B)^{-1} F\left[A \hat{x}(t)+P(t) C^{\top} R(y(t)-C \hat{x}(t)],\right.
$$

which is also optimal for the problem (4). Indeed, this feedback provides $F \frac{d \hat{x}}{d t}=0$, and so, taking into account $\hat{x}(0)=0$, we obtain $F \hat{x}(T)=0$. In fact, we have proved the following

Corollary 1: The control laws (12)-(13) and (14) solve the minimax control problem (4) for $E=0$.

The latter suggests that one may use linear feedback (14) instead of the discontinuous one (12) in order to guarantee the optimal reaching (as close as possible) of the sliding surface in the state space of the original system.

\section{B. The general case: $E \neq 0$ and $D \neq 0$}

Motivated by the results of the previous section, we consider control laws, which guarantee sliding motion on the surface $F \hat{x}=0$ starting from the initial instant of time $t=0$. Evidently, the output-based feedback law (14) as well as the sliding mode algorithm (12), (13) are admissible sub-optimal solutions for the optimization problem (4).

In the case of multiplicative disturbances the matrix $P$ depends on $u$ and formula (7) implies that the volume of the ellipsoid $\left\{e: e^{\top} P^{-1}(t) e \leq 1\right\}$ increases if $\|u(t)\|$ grows. In this case, the chattering phenomenon, introduced by applying the sliding mode control, could imply the significant 
degradation of the control performance compared to the linear control strategy.

We stress that the closed-loop system (1), (6), (7), (14) has an internal dynamic loop : the control signal $u(t)$ depends on $P(t)$ which in turn depends on $u(t) u^{\top}(t)$. The following proposition proves existence and uniqueness of the control.

Proposition 3: Let $F A F_{+}=0$. For any $T>0$ there exists a sufficiently small $\alpha>0$ such that the closed-loop system (1), (6), (7), (14) has the unique bounded solution on $(0, T)$.

The restriction $F A F_{+}=0$ is introduced for simplicity. The same statement can be proven for the general case. Clearly, the control (14) exists on $(0, T), T<+\infty$.

\section{NumeriCAl EXAMPLE}

First, we compare the linear feedback (14) against the sliding mode control (12) for the case of multiplicative disturbances. The case of additive disturbances has been studied in [25]. Consider the system (1) with

$$
\begin{aligned}
& A=\left(\begin{array}{ccc}
0 & 1 & 0.5 \\
-1 & 0 & -0.7 \\
-0.5 & 0.7 & 0
\end{array}\right), B=E=\left(\begin{array}{c}
0.25 \\
0.5 \\
1
\end{array}\right), D=0, \\
& \begin{array}{l}
C=\left(\begin{array}{lll}
1 & 0 & 0
\end{array}\right), \\
F=\left(\begin{array}{lll}
1 & -1 & 1
\end{array}\right), \quad P_{0}=\left(\begin{array}{lll}
1 & 0 & 0 \\
0 & 2 & 0 \\
0 & 0 & 4
\end{array}\right), T=10 . \\
R=10, Q=0, \alpha=1,
\end{array}
\end{aligned}
$$

We also set: $w(t)=\frac{5 \sin (10 t)}{100}, p(t)=5 \cdot 10^{-\frac{3}{2}}$ and take $x_{0}=$ $\left(\begin{array}{lll}1 / 6 & \sqrt{2} / 6 & 2 / 6\end{array}\right)^{\top}$. We used explicit Euler discretization with the time step $10^{-2}$ for the simulations.

Fig. 1 compares the evolution of the sliding variable $\sigma$ corresponding to the linear feedback $u_{e q}$ (14) (red) and the sliding mode control $u_{s m}$ (12), (13) with $\mu=0.5$ (blue). The precision of $u_{e q}$ appears to be better than that of $u_{s m}$. If $\mu$ tends to zero the precision of $u_{s m}$ improves. The simulations exhibit the chattering phenomenon which takes place due to imperfections of numerical discretization of the closed-loop system with the discontinuous control. As noted, we use Euler discretization in order to model sampling effects of digital control devices. Increase of the sampling time leads to further degradation of $u_{s m}$. In practice, non-ideal relays (with delays or/and hysteresis) may provoke additional chattering.

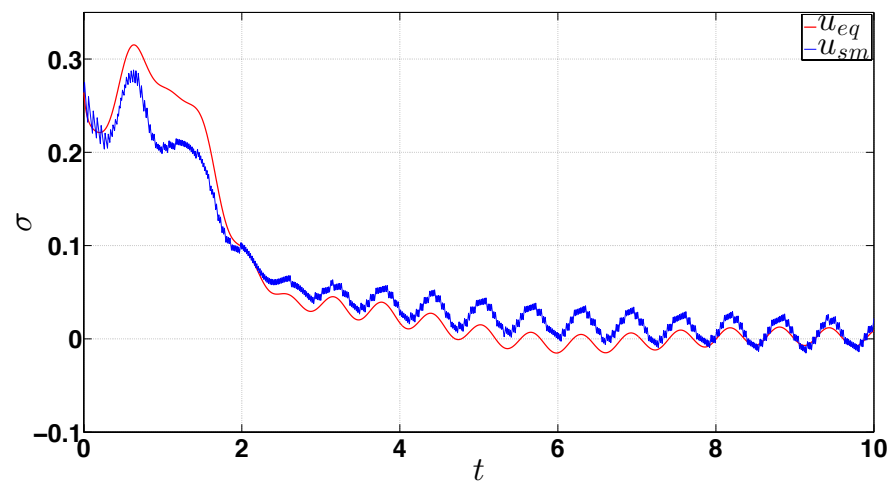

Fig. 1. Comparison of sliding mode and linear control application

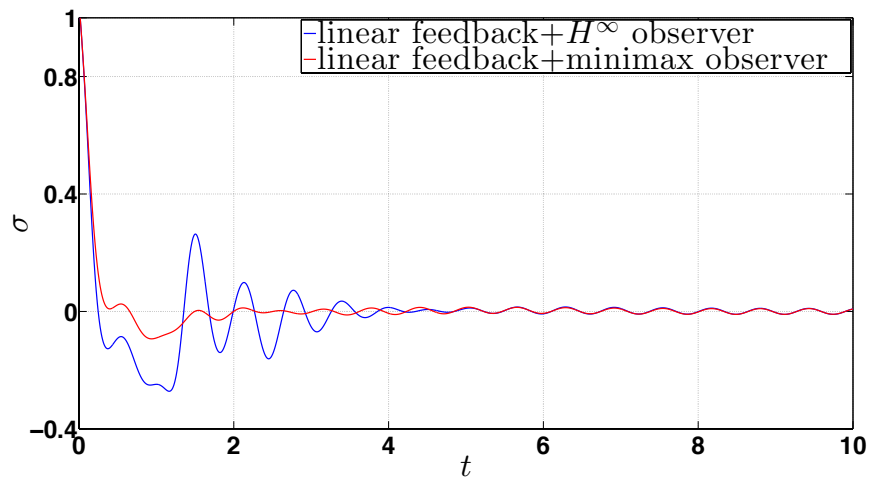

Fig. 2. Comparison of minimax and $H^{\infty}$ observers for linear control (14)

The volume of the ellipsoid (9) defined by the matrix $P(T)$ has decreased by factor $10^{5}$ compared to the initial one, namely $\operatorname{det}(P(T)) / \operatorname{det}\left(P_{0}\right) \approx 10^{-5}$.

Comparison with $H^{\infty}$ observer. Let us now compare the performance of the linear feedback $u_{e q}$ (14) for the minimax and $H^{\infty}$ observers. The $H^{\infty}$ observer also deals with $L_{(0, T)^{-}}^{2}$ disturbances, so it is applicable in our case. The difference between $H^{\infty}$ observer and minimax estimator is in that the latter requires a priori bounds for $L_{(0, T)}^{2}$ disturbances (the ellipsoid (3)) unlike the former. However, prior to implementing $H^{\infty}$ observer one needs to select a so-called "performance parameter" $\gamma \in(0,+\infty)$, which bounds the $H^{\infty}$-norm of the transfer function from the disturbance inputs to the states [12]. In fact, the state equation of $H^{\infty}$ observer coincides with (6), and the gain $P$ solves the Riccati equation (7) with an additional additive term $\frac{1}{\gamma^{2}} P^{2}(t)$ in the right hand side. This immediately implies that the ellipsoidal estimate of the observation error (see, formula (9)) has "larger" shape matrix $P(t)$. In other words, $H^{\infty}$-observers provide more conservative but more robust estimates as opposed to the sharp (less conservative) estimate (9) of the minimax observer. In addition, $H^{\infty}$ observer performance is quite sensitive to the choice of $\gamma$, as it needs to be chosen so that the difference $\frac{1}{\gamma^{2}} P^{2}(t)-P(t) C^{\top} R C P(t)$ does not grow too fast. The figure 2 compares the evolution of the sliding variable $\sigma$ for the minimax and $H^{\infty}$ observers (with $\gamma=2.75$, and $\left.x_{0}=\frac{2}{3}(1, \sqrt{2}, 2)^{\top}\right)$.

\section{CONCLUSION}

The paper studies a problem of sliding mode control design for linear time invariant systems with incomplete and noisy measurements of the output and additive/multiplicative exogenous disturbances. The key outcomes of the presented study for the case of additive disturbances are as follows:

- The conventional sliding mode control provides one of many possible solutions of the problem of optimal reaching of the sliding surface (4).

- Sliding motion of the observer variable may be attained using a linear optimal feedback (14). In fact, the latter coincides with the equivalent control of the sliding mode system. Since all the parameters of the observer state equation are known, this equivalent control can be always found explicitly!

Despite of the fact that both solutions (sliding mode and linear feedback) theoretically provide the same precision, the chat- 
tering phenomenon significantly decreases the performance of the sliding mode control. In the case of multiplicative disturbances, the estimation precision depends on the control magnitude implying the additional degradation of the performance. This fact is confirmed by numerical simulations.

We conclude that the linear feedback control provides an optimal solution of output-based sliding mode control design problem for linear systems with $L^{2}$ noises and additive disturbances. This fact together with practical issues encountered while applying the discontinuous sliding mode output-based control under the same assumptions suggests that the conventional sliding mode control methodology requires a radical rethink for linear systems. Perhaps, the conventional sliding mode control becomes the unique optimal solution of (4) provided the class of noises and disturbances is restricted to $L^{\infty}$. The rigorous study of this conjecture is left for the future research.

\section{APPENDIX}

\section{A. Proof of Proposition 1}

To prove the proposition we first rewrite the minimax estimation problem (see definition 1) in the operator form. As noted below (see proof of proposition 2) to estimate $l_{0}^{\top} x(T)$ one needs to estimate just the "noisy part" of $x(T)$, namely $x_{g}(T)$ defined by the 2 nd equation of (15). Let us recall that the latter induces a linear bounded integral operator $W: H_{1} \rightarrow H_{2}$. Indeed, define $H_{1}:=L_{(0, T)}^{2} \times L_{(0, T)}^{2} \times \mathbb{R}^{n}$, $H_{2}:=L_{(0, T)}^{2}, q:=\left(p, d, x_{0}\right)$ and set $W q:=e^{A t} x_{0}+$ $\int_{0}^{t} e^{A(t-s)}(D d(s)+p(s) E u(t)) d s$ for any $q \in H_{1}$. We also note that $l_{0}^{\top} x_{g}(T)=x_{0}^{\top} e^{A^{\top} T} l+\int_{0}^{T} d^{\top}(s) e^{A^{\top}(T-s)} D^{\top} l+$ $p(s)(E u(s))^{\top} e^{A^{\top}(T-s)} l d s:=\ell(q)$, and $\ell(q)$ is a linear continuous functional over $H_{1}$. In other words, computing the minimax estimate of $l_{0}^{\top} x_{g}(T)$ given $y_{g} \in H_{2}$ (see (16)) and assuming that (3) holds true, is equivalent to computing the minimax estimate of $\ell(q)$ provided $y_{g}=C W q+w$ and $(q, w) \in \mathscr{E}$ where $\mathscr{E}:=\{(q, w): \rho(q, w) \leq 1\}$ and $\rho(q, w):=\left\|\left(\alpha^{-\frac{1}{2}} p, Q^{\frac{1}{2}} d, P_{0}^{-\frac{1}{2}} x_{0}\right)\right\|_{H_{1}}^{2}+\left\|R^{\frac{1}{2}} w\right\|_{H_{2}}^{2}$.

Let us now prove the proposition. Our proof relies upon Banach separation principle. Let $\mathscr{G}\left(H_{i}\right)$ denote the set of all continuous functionals $f: H_{i} \rightarrow \mathbb{R}$ and let $H_{i}^{\star}$ denote a subset of $\mathscr{G}\left(H_{i}\right)$ composed of all linear functionals, $i=1,2$. We claim that:

$\inf _{f \in \mathscr{G}\left(H_{2}\right)} \sup _{(q, w) \in \mathscr{E}}\left|\ell(q)-f\left(y_{g}\right)\right|=\inf _{V \in H_{2}^{\star}} \sup _{(q, w) \in \mathscr{E}}\left|\ell(q)-V\left(y_{g}\right)\right|$.

Indeed, the inequality $\inf _{f \in \mathscr{G}\left(H_{2}\right)} \sup _{(q, w) \in \mathscr{E}} \leq \inf _{V \in H_{2}^{\star}} \sup _{(q, w) \in \mathscr{E}}$ is obvious as $H_{2}^{\star} \subset \mathscr{G}\left(H_{2}\right)$. Let us prove that for any $\ell \in H_{1}^{\star}$ there exists $q_{0}$ and $V \in H_{2}^{\star}$ such that

$\sup _{(q, w) \in \mathscr{E}}\left|\ell(q)-V\left(y_{g}\right)\right| \leq \ell\left(q_{0}\right) \leq \inf _{f \in \mathscr{G}\left(H_{2}\right)} \sup _{(q, w) \in \mathscr{E}}\left|\ell(q)-f\left(y_{g}\right)\right|(*)$

Indeed, define $\mathscr{E}_{0}:=\{(q, w) \in \mathscr{E}: 0=C W q+w\}$. Clearly, $\mathscr{E}_{0}$ is a closed convex bounded set and so $\sup _{\mathscr{E}_{0}} \ell(q)=\ell\left(q_{0}\right)<$ $+\infty$ for some $\left(q_{0}, w_{0}\right) \in \mathscr{E}_{0}$. Note that $(q, w) \in \mathscr{E}_{0}$ implies $-(q, w) \in \mathscr{E}_{0}$. Hence, $\inf _{\mathscr{E}_{0}} \ell(q)=-\ell\left(q_{0}\right)$. Now, define $Y:=$ $\left\{\left(\ell(q), y_{g}\right): y_{g}=C W q+w,(q, w) \in \mathscr{E}\right\}$. Clearly, $\left( \pm \ell\left(q_{0}\right), 0\right)$ are extreme points of $Y$ (as $\pm q_{0}$ is the maximizer/minimizer of $\ell$ over $\mathscr{E}_{0}$ ). Since the set $Y$ is convex and $m \in Y$ implies $-m \in Y$, it follows that there exist a scalar $s>0$ and a linear functional $V_{0} \in H_{2}^{\star}$ such that the hyperplanes $H_{ \pm}:=$ $\left\{\left(\ell(q), y_{g}\right): s\left(\ell(q) \pm \ell\left(q_{0}\right)\right)+V_{0}\left(y_{g}\right)=0\right\}$ are supporting for the set $Y$, i.e., $s \ell\left(q-q_{0}\right)+V_{0}\left(y_{g}\right) \leq 0$ and $s \ell\left(q+q_{0}\right)+$ $V_{0}\left(y_{g}\right) \geq 0$ for all $\left(\ell(q), y_{g}\right) \in Y$. Set $V\left(y_{g}\right):=s^{-1} V_{0}\left(y_{g}\right)$. We have: $\left|\ell(q)+V\left(y_{g}\right)\right| \leq \ell\left(q_{0}\right)$ for all $\left(\ell(q), y_{g}\right) \in Y$ so that $\sup _{(q, w) \in \mathscr{E}}\left|\ell(q)+V\left(y_{g}\right)\right| \leq \ell\left(q_{0}\right) . \quad(* *)$

On the other hand, we note that $0 \leq 2 \ell\left(q_{0}\right)=\ell\left(q_{0}\right)-V\left(y_{g}\right)+$ $V\left(y_{g}\right)-\ell\left(-q_{0}\right) \leq\left|\ell\left(q_{0}\right)-V\left(y_{g}\right)\right|+\left|V\left(y_{g}\right)-\ell\left(-q_{0}\right)\right| \leq$ $2 \sup _{(q, w) \in \mathscr{E}}\left|\ell(q)-V\left(y_{g}\right)\right|$. The latter and $(* *)$ prove $(*)$. This completes the proof of the proposition's claim.

\section{B. Proof of Proposition 2}

Let $x_{u}, x_{g}$ denote the solutions of the following ODEs:

$$
\begin{array}{ll}
\frac{d x_{u}(t)}{d t}=A x_{u}(t)+B u(t), & x_{u}(0)=0, \\
\frac{d x_{g}(t)}{d t}=A x_{g}(t)+D d(t)+p(t) E u(t), & x_{g}(0)=x_{0} .
\end{array}
$$

Then, clearly, $x(t)=x_{u}(t)+x_{g}(t)$ and

$$
y_{g}(t):=y(t)-C x_{u}(t)=C x_{g}(t)+w(t) .
$$

The function $x_{g}$ may be considered as a "noisy" part of $x$ corresponding to disturbances from the ellipsoid (3). In contrast, $x_{u}$ represents the "mean" value of $x$ which corresponds to the case: $x_{0}=0, p=0$ and $d=0$. Let us estimate $x_{g}$. To do so we use classical results on minimax filters, namely it can be demonstrated (see for instance [9]) that the minimax estimate of $l^{\top} x_{g}\left(t^{*}\right)$ is given by $\widehat{\mathcal{U}}_{l}\left(y_{g}\right)=l^{\top} \hat{x}_{g}\left(t^{*}\right)$ and the minimax estimation error can be represented as $\hat{\sigma}\left(l, t^{*}\right)=\left(l^{\top} P\left(t^{*}\right) l\right)^{\frac{1}{2}}$ provided $y_{g}$ is defined by (16) and

$$
\left\{\begin{array}{l}
\frac{d \hat{x}_{g}(t)}{d t}=A \hat{x}_{g}(t)+P(t) C^{\top} R\left(y_{g}(t)-C \hat{x}_{g}(t)\right), \\
\hat{x}_{g}(0)=0 .
\end{array}\right.
$$

Now we recall that $x\left(t^{*}\right)=x_{u}\left(t^{*}\right)+x_{g}\left(t^{*}\right)$ and so $x\left(t^{*}\right)=$ $x_{u}\left(t^{*}\right)+\hat{x}_{g}\left(t^{*}\right)+e\left(t^{*}\right)$, where $e\left(t^{*}\right)=x_{g}\left(t^{*}\right)-\hat{x}_{g}\left(t^{*}\right)$. Then, by definition of the minimax error we get: $l^{\top} e\left(t^{*}\right) \leq$ $\left(l^{\top} P\left(t^{*}\right) l\right)^{\frac{1}{2}}$. Define $\hat{x}:=x_{u}+\hat{x}_{g}$. Then it is straightforward to check that $\hat{x}$ satisfies (6) and the error estimate holds.

\section{Proof of Theorem 1}

Let $J(u)$ be the value of the functional defined by (4). Having in mind (15) we write:

$$
\begin{gathered}
\sigma(T)=F x_{u}(T)+F x_{g}(T)=F x_{u}(T)+F \hat{x}_{g}(T)+F e(T) \\
=F \hat{x}(T)+F e(T)=\hat{\sigma}(T)+F e(T),
\end{gathered}
$$

where $e(T) \in \mathcal{E}=\left\{z \in \mathbb{R}^{n}: z^{T} P^{-1}(T) z \leq 1\right\}$. Since $\hat{x}+\mathcal{E}$ is the reachability set of the system (15) it follows that $\hat{x}-x \in \mathscr{E}$ if and only if $x$ solves equation (1) provided $x_{0}$, $p, d$ and $v$ verify (3) and, hence, we obtain:

$$
J(u)=\sup _{e \in \mathcal{E}}\left\|F x_{u}(T)+F \hat{x}_{g}(T)+F e\right\|
$$

If $E=0$ then $x_{g}$ and $P(T)$ do not depend on $u$, so for any $u: \hat{\sigma}(T)=0$ we have $J(u)=\sup _{e \in \mathcal{E}}\|F e\|$. We 
now note that $\sup _{e \in \mathcal{E}}\|\eta+F e\|=\sup _{e \in \mathcal{E}} \sup _{l:\|l\|=1} l^{\top}(\eta+$ $F e)=\sup _{l:\|l\|=1}\left(l^{\top} \eta+\sup _{e \in \mathcal{E}} l^{\top} F e\right)=\sup _{l:\|l\|=1}\left(l^{\top} \eta+\right.$ $\left.\left((P(T) F l)^{\top} F l\right)^{\frac{1}{2}}\right) \geq \sup _{l:\|l\|=1}\left(\left((P(T) F l)^{\top} F l\right)^{\frac{1}{2}}\right)=$ $\sup _{e \in \mathcal{E}}\|F e\|$, where the inequality used in the previous line follows from the fact that the sphere $l:\|l\|=1$ is symmetric w.r.t. 0. Hence, $\sup _{e \in \mathcal{E}}\|F e\| \leq \sup _{e \in \mathcal{E}}\|\eta+F e\|, \forall \eta \in \mathbb{R}^{m}$.

\section{Proof of Proposition 3}

I. We prove this proposition under assumption that $F A F_{+}=0$, where $F_{+}=I-B(F B)^{-1} F$ is the projector onto the hyperplane $F \hat{x}=0$. The general case can be treated in the similar way.

Substitution of (14) into (6) gives

$\frac{d \hat{x}(t)}{d t}=F_{+}\left(A \hat{x}(t)+P(t) C^{\top} R[C e(t)+w(t)]\right), \quad \hat{x}(0)=0$.

Hence, we derive

$$
\hat{x}(t)=\int_{0}^{t} e^{F_{+} A(t-s)} F_{+} P(s) C^{\top} R[C e(s)+w(s)] d s .
$$

and, due to $F A F_{+}=0$,

$$
F A \hat{x}(t)=F A \int_{0}^{t} e^{F_{+} A(t-s)} F_{+} P(s) C^{\top} R[C e(s)+w(s)] d s=0 .
$$

We proved that $u(t)=(F B)^{-1} F P(t) C^{\top} R[C e(t)+w(t)]$.

$$
\text { II. Denoting } L(t)=(F B)^{-1} F P(t) C^{\top} R\left(C P^{\frac{1}{2}}(t) R^{-\frac{1}{2}}\right) \text { we }
$$

get $u(t) u^{\top}(t)=L(t)\left(\begin{array}{c}P^{-\frac{1}{2}}(t) e(t) \\ R^{\frac{1}{2}} w(t)\end{array}\right)\left(\begin{array}{c}P^{-\frac{1}{2}}(t) e(t) \\ R^{\frac{1}{2}} w(t)\end{array}\right)^{\top} L^{\top}(t)$.

Recalling that $v v^{\top} \leq v^{\top} v I_{n}$ for any $v \in \mathbb{R}^{n}$ and taking into account $e^{\top}(t) P^{-1}(t) e(t) \leq 1$ we derive:

$$
\begin{gathered}
u(t) u^{\top}(t) \leq L(t)\left(\begin{array}{c}
P^{-\frac{1}{2}}(t) e(t) \\
R^{\frac{1}{2}} w(t)
\end{array}\right)^{\top}\left(\begin{array}{c}
P^{-\frac{1}{2}}(t) e(t) \\
R^{\frac{1}{2}} w(t)
\end{array}\right) L^{\top}(t) \leq \\
\left(1+w^{\top}(t) R w(t)\right) L(t) L^{\top}(t)
\end{gathered}
$$

III. Let us consider the closed loop system

$$
\begin{aligned}
\dot{P}(t) & =A P(t)+P(t) A^{\top}+D Q^{-1} D^{\top}-P(t) N P(t)+\alpha u(t) u^{\top}(t), \\
\dot{e}(t) & =A e(t)-P(t) C^{\top} R(C e(t)+w(t))+p(t) E u(t)+D g(t), \\
u(t) & =(F B)^{-1} F P(t) C^{\top} R[C e(t)+w(t)] .
\end{aligned}
$$

with $P(0)=P_{0}, e(0)^{T} P_{0}^{-1} e(0) \leq 1$.

Let us denote the right-hand side of the considered system by $R H S(t, e, P, \alpha)$, where $e, P$ are components of the extended state vector and $\alpha$ considered as a constant parameter.

In order to prove the existence and uniqueness of the solution of this equation for $t \in[0, T]$ and for sufficiently small $\alpha>0$, we can use the theorem on continuous dependence on the parameter (see, for example, [4], Chapter 2, Theorem 4.2.). All conditions of the aforementioned theorem hold for the considered system. Indeed, for $\alpha=0$ the system has a unique solution. The righthand side $R H S$ is a continuous function with respect to $e, P, \alpha$ for any fixed $t$. Since for any $h \in \mathbb{R}^{n}$ we have $\left|h^{\top} p(t) \Xi w(t)\right| \leq p^{2}(t) h^{\top} \Xi R^{-1} \Xi h+w^{\top}(t) R w(t)$, where $\Xi=E(F B)^{-1} F P(t) C^{\top} R$, then taking into account the estimate of $u^{\top} u$ obtained above we derive that $R H S$ is measurable with respect to $t$ for any fixed $e, P, \alpha$ and its norm is bounded (locally) by an integrable function $m$ : $|R H S(t, e, P)|<m(t)$ for $t \in[0, T]$.

\section{REFERENCES}

[1] V. G. Boltyanski and A. Poznyak. The Robust Maximum Principle: Theory and Applications. Birkhauser Basel, 2012.

[2] F. Castanos and L. Fridman. Analysis and design of integral sliding manifolds for systems with unmatched perturbations. IEEE Transactions on Automatic Control, 51(5):853-858, 2006.

[3] F.L. Chernousko. State Estimation for Dynamic Systems. Boca Raton, FL: CRC, 1994.

[4] E. A. Coddington and N. Levinson. Theory of Ordinary Differential Equations. New York, McGraw-Hill, 1955.

[5] J. Davila, L. Fridman, and Levant A. Second-order sliding-mode observer for mechanical systems. IEEE Transactions on Automatic Control, 50(11):1785-1789, 2005.

[6] C. Edwards, A. Akoachere, and S. K. Spurgeon. Sliding-mode output feedback controller design using linear matrix inequalities. IEEE Transactions on Automatic Control, 46(1):115-119, 2001.

[7] C. Edwards and S. Spurgeon. Sliding Mode Control: Theory And Applications. CRC Press., 1998.

[8] C. Edwards and S. K. Spurgeon. Sliding mode stabilization of uncertain systems using only output information. Int. J. of Control, 62(5):1129$1144,1995$.

[9] Arthur J. Krener. Kalman-Bucy and minimax filtering. IEEE Trans. on Autom. Control, 25(2):291-292, Apr 1980.

[10] A. Kurzhanski and I. Valyi. Ellipsoidal calculus for estimation and control. Birkhauser Boston Inc., Boston, MA, 1997.

[11] M. Milanese and R. Tempo. Optimal algorithms theory for robust estimation and prediction. IEEE Tran. Autom. Cont., 30(8):730-738, 1985.

[12] K. M. Nagpal and P. P. Khargonekar. Filtering and smoothing in $h^{\infty}$ setting. IEEE Transactions on Automatic Control, 36(2):151-166, 1991.

[13] A. Polyakov. Nonlinear feedback design for fixed-time stabilization of linear control systems. IEEE Trans. Autom. Cont., 57(8):2106-2110, 2012.

[14] A. Polyakov and L. Fridman. Stability notions and lyapunov functions for sliding mode control systems. Journal of The Frankin Institute, 351(4):1831-1865, 2014.

[15] A. Polyakov and A. Poznyak. Invariant ellipsoid method for minimization of unmatched disturbance effects in sliding mode control. Automatica, 47(7):1450-1454, 2011.

[16] A.S. Poznyak. Variable Structure Systems: from Principles to Implementation, volume 66 of IEE Control Series, chapter Deterministic output noise effects in sliding mode observation, pages 45-78. The IET, London, UK, 2004.

[17] A.S. Poznyak. Advanced Mathematical Tools for Automatic Control Engineers: Deterministic Techniques. Elsevier, 2008.

[18] Y. Shtessel, C. Edwards, L. Fridman, and A. Levant. Sliding Mode Control and Observation. Birkhauser, 2014.

[19] V. I. Utkin. Sliding Modes in Control and Optimization. Springer Verlag, Berlin, 1992.

[20] V.I. Utkin. Variable structure systems with sliding modes. IEEE Transactions on Automatic Control, 22(2):212 - 222, 1977.

[21] V.I. Utkin, J. Guldner, and J. Shi. Sliding Mode Control in ElectroMechanical Systems. CRC Press., 2009.

[22] S. Zhuk. Estimation of the states of a dynamical system described by linear equations with unknown parameters. Ukrainian Math. J., 61(2):214-235, 2009

[23] S. Zhuk. Kalman duality principle for a class of ill-posed minimax control problems with linear differential-algebraic constraints. Applied Mathematics and Optimisation, 68(2):289-309, 2013.

[24] S. Zhuk and M. Petreczky. Infinite horizon control and minimax observer design for linear daes. In Proc. 52nd IEEE Conference on Decision and Control, 2013.

[25] S. Zhuk and A. Polyakov. On output-based sliding mode control design using minimax observer. In 13th Workshop on Variable Structure Systems, Nantes, France, 2014.

[26] S. Zhuk and A. Polyakov. On output-based sliding mode control design using minimax observer. In European Control Conference, Linz, Austria, 2015. 